\section{Generalized mid dermal elastolysis}

\author{
Maria João Cruz, Ana Margarida Barros, \\ Filomena Azevedo \\ Department of Dermatology and \\ Venereology, Hospital de São João, E.P.E. \\ Porto, Portugal
}

\begin{abstract}
Mid-dermal elastolysis (MDE) is a rare skin disorder clinically characterized by the appearance of diffuse fine wrinkling, most often of the trunk and arms. This entity is distinguished from other elastolytic disorders by its characteristic selective loss of elastic fibers of the mid dermis. The aetiopathogenesis of the disease is still unclear as well as the effective treatment. Half of the cases described in the literature are associated with ultraviolet radiation exposure. Other reported triggering conditions such as urticaria, eczema and granuloma annulare suggests different eliciting inflammatory pathways. The authors describe the case of a 38-year-old woman who developed an urticarial eruption during months which progressed to generalized and severe fine wrinkling.
\end{abstract}

\section{Introduction}

Mid-dermal elastolysis (MDE) is a rare idiopathic skin disorder of the elastic tissue first described in 1977 by Shelly and Wood. This entity falls into a spectrum of acquired elastolytic disorders that also includes cutis laxa acquisita, anetoderma, pseudoxanthoma elasticum papillary dermal elastolysis and linear focal elastolysis. It is distinguished from those conditions by its characteristic selective loss of elastic fibers of the mid dermis. ${ }^{2,3}$ The clinical appearance is variable and may include patches of well-circumscribed fine wrinkles, perifollicular papular protusions and/or inflammatory changes such as reticular erythema. ${ }^{4}$ The most frequently affected skin sites are the trunk, the neck and the arms. The aetiopathogenesis of the disease is still unclear, however several theories have been proposed to justify the destruction of the elastic fibers, but the actinic damage and inflammation gather together more consensus. ${ }^{2,4,5}$

\section{Case Report}

We describe the case of a 38-year-old woman, otherwise healthy, that was observed in our department due to a 9-month history of urticarial eruption that started on the thorax and flexor surface of the arms and had a descendent progression, evolving the abdomen and thighs. The eruption progressively subsided, giving place to a symmetrically fine wrinkling parallel to skin cleavage lines (Figure 1). Skin biopsies in urticarial lesions and in wrinkling areas showed a perivascular and interstitial lymphocytic inflammatory infiltrate that surrounded the dermal vessels (Figure 2). Stains for elastic fibers (orsein) revealed a band-like loss of elastic fibers limited to the mid dermis (Figure 3). The patient revealed only sporadic sun exposure and there was no family history of similar skin changes. No drugs, alcohol or smoking habits were recorded. She was submitted to multiple treatment trials along the three years of follow-up. Initially, as the urticarial/inflammatory lesions predominated, the patient was treated with short courses of topical betametsone $1 \mathrm{mg} / \mathrm{g}$ and oral prednisolone $0.75 \mathrm{mg} / \mathrm{kg}$ which was gradually reduced and interrupted after 3 months due to the lack of results. Due to the progression of the lesions to extensive wrinkling, corticosteroid treatment was followed by retinoids, including topical tretinoin $(0.5 \mathrm{mg} / \mathrm{g})$ and oral isotretinoin (started at the dose of $40 \mathrm{mg} /$ day which was gradually reduced to $10 \mathrm{mg} / \mathrm{day}$ ). None of those prevented the progression of wrinkling, however the patient referred partial improvement of the skin texture. In the last visit was notorious the widespread wrinkling, sparing only the face, hands, lower legs and feet (Figures 4A and 4B). The skin biopsy at this time showed total loss of elastic fibers in the mid dermis but no perivascular inflammatory infiltrate. All the study performed over time in order to detect precipitating factors or disorders was normal or negative including renal, hepatic and thyroid function, immunological and coagulation tests, viral markers (HIV, HBV and HCV), tumoral markers, thorax $\mathrm{X}$-Ray, abdominal and thyroid ecographies, thoracic and abdominal tomography scans, upper gastrointestinal endoscopy and colonoscopy.

\section{Discussion}

MDE is a rare acquired skin condition of the elastic tissue predominantly affecting young women. Only about 80 cases have been reported in literature and, to date, the precise pathogenesis of elastolysis has not been elu-
Correspondence: Maria João Cruz, Department of Dermatology and Venereology, Hospital São João, EPE, Alameda Prof. Hernani Monteiro, 4202-451 Porto, Portugal.

Tel. +351.225.512.193 - E-mail: mjmc@live.com.pt

Key words: mid dermal elastolysis, disorders of elactic fibers, elastophagocytosis.

Received for publication: 20 July 2011.

Revision received: 30 August 2011.

Accepted for publication: 24 October 2011.

This work is licensed under a Creative Commons Attribution NonCommercial 3.0 License (CC BYNC 3.0).

(C) Copyright M.J. Cruz et al., 2011

Licensee PAGEPress, Italy

Dermatology Reports 2011; 3:e52

doi:10.4081/dr.2011.e52

cidated. ${ }^{2,4}$ In half of the cases remarkable ultraviolet exposure (sun, phototherapy or sun beds) was recognized as the trigger of the disease ${ }^{4,6}$ Cho and co-workers observed that intense sunlight as well as heat significantly increase macrophage dermal infiltration inducing their proinflammatory cytokine production, raise the levels of collagenolytic enzymes, including matrix metalloproteinases (MMP-1 and MMP-9) and decreased procollagen type I expression which can be responsible, at least in part, for the degradation of elastic fibers. ${ }^{78}$ However, the paucity of actinic damage in MDE lesions and the sparing of heavily light exposed sites (such as face and back of the hands) in those patients, raise the doubt about the true pathogenic role of ultraviolet radiation on this condition. Moreover, remains to be explained why fiber destruction is limited to the mid dermis. Nowadays, many authors tend to agree with the idea that optical radiation is an unspecific inflammatory trigger of MDE. ${ }^{2,45}$ The sporadic association with other common inflammatory conditions including urticaria, eczema, granuloma annulare, pityriasis rosea, Sweet syndrome, phototoxic dermatitis, guttate psoriasis, rheumatoid arthritis, Hashimoto's thyroiditis may be merely coincidental or, most probably, suggest different eliciting inflammatory pathways by unspecific inflammatory triggers. ${ }^{2,4,6}$

In our patient, the lack of intense sun exposure and the sparing of the face, dorsum of the hands and lower legs, play against the ultraviolet exposure as the main pathogenic factor. Nevertheless, the urticarial lesions and the perivascular inflammatory infiltrate in the histological examination of the early lesions clearly suggest a post-inflammatory etiology. However, no other disease or possible pathogenic factor was identified as trigger 


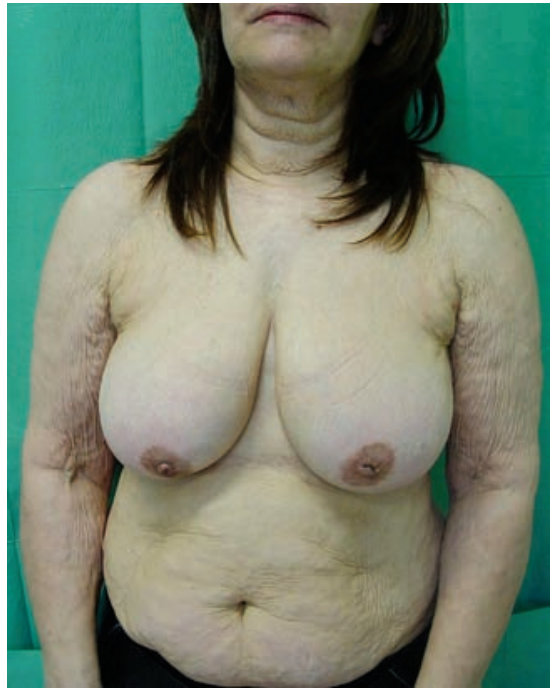

Figure 1. Fine wrinkling parallel to skin cleavage lines, with symmetrical distribution.

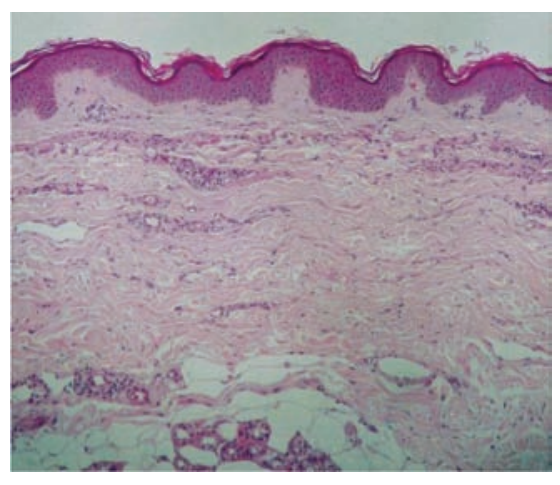

Figure 2. Skin biopsies showed a perivascular and interstitial lymphocytic inflammatory infiltrate that surrounded the dermal vessels.

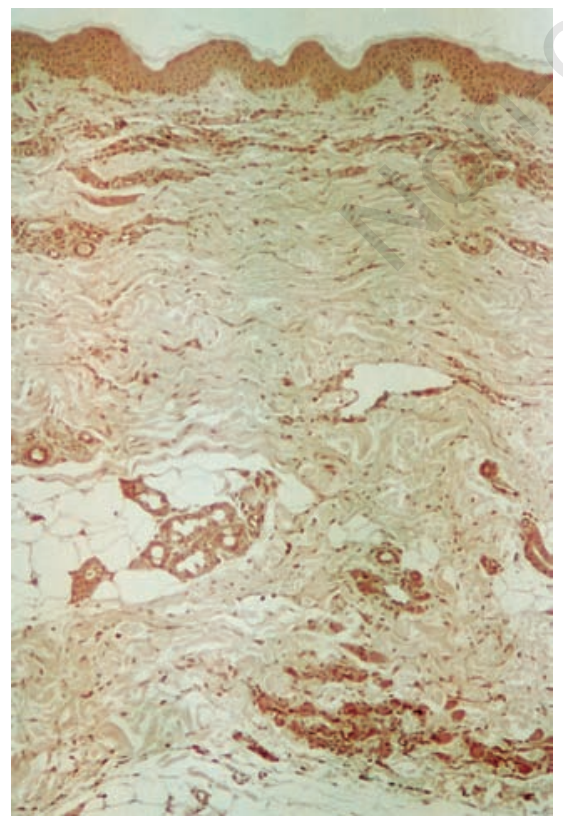

Figure 3. Stains for elastic fibers (orsein) revealed a band-like loss of elastic fibers limited to the mid dermis.

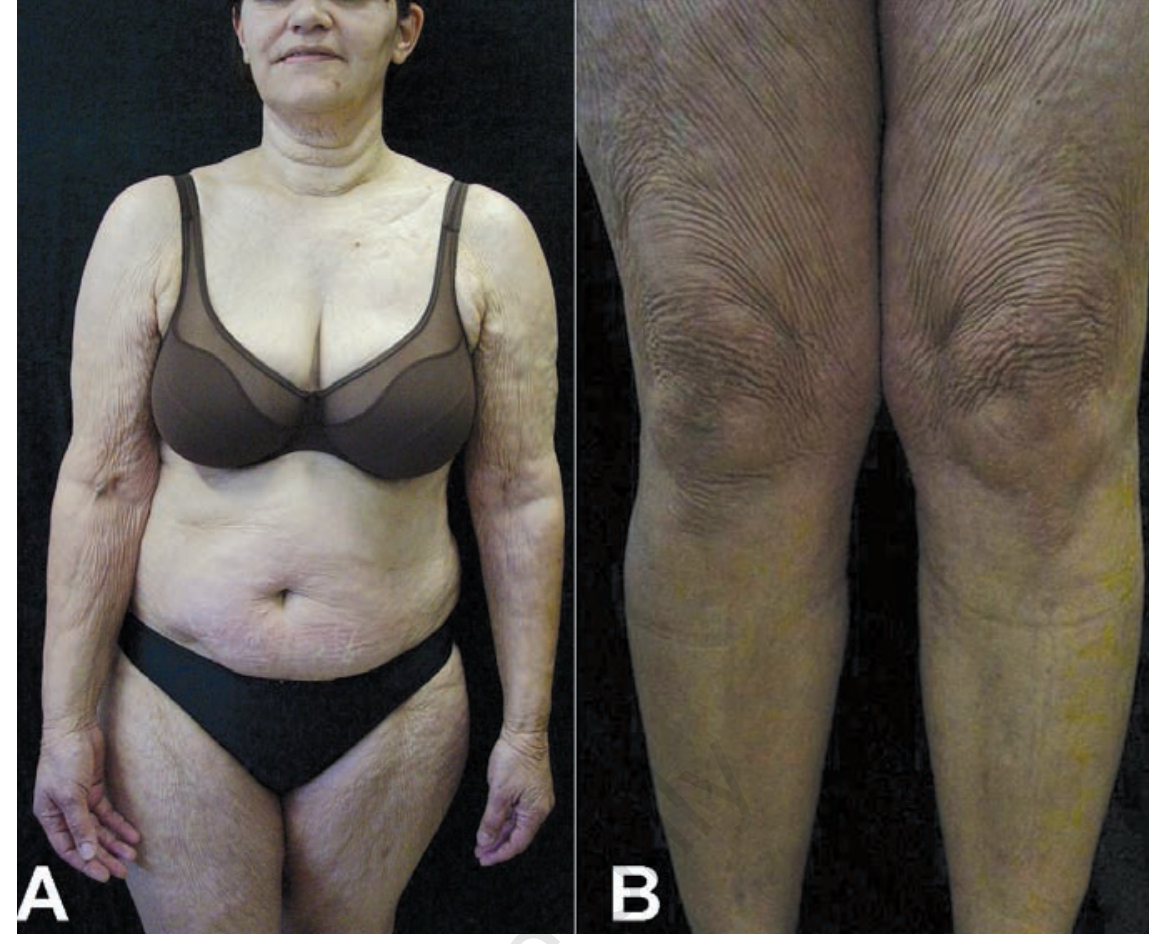

Figure 4. Progressive worsening with widespread wrinkling, sparing only the face, hands, lower legs and feet.

of inflammation. ${ }^{2,4,5,9}$ To date, no definitive therapy exists. Vitamin E, dapsone, clofazimine, chloroquine, colchicine, topical and systemic steroids have been tried but no clear benefit was observed with any of those drugs alone or in combination. Despite knowing that probably oral and topical retinoid wouldn't change the course of the disease, this therapeutic option, risks and (uncertain) benefits were discussed with the patient who decided to maintain the treatment once she was feeling a slight improvement and no other effective treatment is currently available. Future therapeutic approaches in MDE patients should focus on agents that are able to block increased elastase activity and induce elastin synthesis. ${ }^{2,4,5}$

Analysis of a greater number of cases will shed more light on this disease.

\section{References}

1. Shelley WB, Wood MG. Wrinkles due to idiopathic loss of mid-dermal elastic tissue. Br J Dermatol 1977;97:441-5.

2. Patroi I, Annessi G, Girolomoni G. Mid-dermal elastolysis: a clinical, histologic, and immunohistochemical study of 11 patients. J Am Acad Dermatol 2003;48:
$846-51$.

3. Lewis KG, Bercovitch L, Dill SW, RobinsonBostom L. Acquired disorders of elastic tissue: Part II. decreased elastic tissue. J Am Acad Dermatol 2004;51:165-85.

4. Gambichler T. Mid-dermal elastolysis revisited. Arch Dermatol Res 2010;302:8593.

5. Streams BN, Williams JM, Moschella SM. Mid-dermal elastolysis. Cutis 2003;71: 3124.

6. Vatve M, Morton R, Bilsland D.A case of mid-dermal elastolysis after narrowband ultraviolet B phototherapy. Clin Exp Dermatol 2009;34:263-4.

7. Cho S, Lee MJ, Kim MS, et al. Infrared plus visible light and heat from natural sunlight participate in the expression of MMPs and type I procollagen as well as infiltration of inflammatory cell in human skin in vivo. $\mathrm{J}$ Dermatol Sci 2008;50:123-33.

8. Sanyal S, Hejmadi R, Taibjee SM. Granuloma annulare resolving with features of mid-dermal elastolysis. Clin Exp Dermatol 2009;34:e1017-8.

9. Gambichler T, Stücker M, Kreuter A, et al. Expression of extracellular matrix proteins in reticular variant of mid-dermal elastolysis. J Eur Acad Dermatol Venereol 2010;24:1481-4 